\title{
Aprendiendo a ver y dibujar
}

\author{
Francisco CABALlero RodríGUez \\ Universidad de Granada \\ francis@ugr.es
}

\begin{abstract}
Resumen:
La interacción entre las tecnologías aplicadas desarrolladas en el campo de la visión computacional, la percepción visual humana estudiada desde las teorías de la Gestalt y la revisión de la obra de los artistas más significativos, se retroalimentan entre sí y sirven de base para la formulación de estrategias que ayudan en la comprensión de los fenómenos perceptuales a la hora de enfrentarse al dibujo de observación y a su aprendizaje.

Uno de los objetivos fundamentales de este trabajo se centra precisamente en dar visibilidad a los procesos intelectuales que intervienen en los procesos de visión-percepción-representación que están presentes en el software de tratamiento de la imagen digital, favoreciendo notablemente los resultados obtenidos en la práctica experimental del Dibujo.
\end{abstract}

Palabras clave: Dibujo; Percepción visual; Tecnologías aplicadas al Dibujo

\section{Learning to see and draw}

\begin{abstract}
:
The interaction among applied technologies developed into the area of computer vision, human visual perception studied from Gestalt theories, and reviewing on the work of the most significant artists, feed back each other and provide a basis for the formulation of strategies that help in understanding the perceptual phenomena when we face the observational drawing and its learning.

One of the primary objectives of this work focuses precisely on giving visibility to the intellectual processes involved in the processes of vision-perception-representation that are present in the software for digital image processing, notably encouraging results obtained in the experimental practice of drawing.
\end{abstract}

Key words: Drawing,; Visual perception; Applied technologies to drawing

\section{Referencia normalizada:}

Caballero Rodríguez, F. (2014): Aprendiendo a ver y dibujar. Historia y Comunicación Social. Vol. 19. Núm. Especial Marzo. Págs. 551-564.

Sumario: 1. Introducción, 2. Metodología, 2.1 Planteamiento metodológico, 2.2 Fases de la investigación, 2.2.1 Identificación de dificultades, 2.2.2 Estudio de las fuentes bibliográficas clásicas y comtemporáneas, 2.2.3 Los artístas clásicos y contemporáneos relacionados con el tema en cuestión, 2.2.4 Elementos que intervienen en los procesos de visión-percepción-representación, 2.2.5 Uso de estrategias asociadas a tecnologías de la imagen, 2.2.5.1 Bordes de una imagen, 3. Resultados, 4. Conclusiones, 5. Bibliografía 


\section{Introducción}

Los inicios del aprendizaje del dibujo en las Facultades de Bellas Artes son un campo donde se gesta gran parte del conocimiento posterior y su desarrollo a lo largo del periodo de formación y de toda la vida y experiencia creadora. Este artículo muestra una síntesis de la experiencia llevada a cabo con estudiantes de primer curso de los estudios de Bellas Artes.

Dibujar es conocer, y este proceso nos ayuda a comprender el mundo. El dibujo de observación persigue la reproducción de una escena real mediante el artificio de la representación. Para ello hemos huir de las representaciones esquemáticas o simbólicas y hemos de encontrar un lenguaje que haga resultar creíble la imagen proyectada mediante uso de leyes, reglas y convenciones que soporten el simulacro sin que resulten meramente anecdóticas.

Estas primeras aproximaciones a la práctica del dibujo de observación son para muchos de los estudiantes su primera inmersión en un mundo en el que comienzan a descubrir su componente intelectual. Descubren que la clave no está en la "buena mano", y que todo proceso de percepción activa implica un minucioso análisis del mundo que les rodea.

Las distintas formas de abordar tanto la extracción de la información visual en el acto de dibujar, como la ejecución o puesta en escena de los recursos que articulan la imagen sobre el papel, conforman parte de las estrategias de la enseñanza-aprendizaje del dibujo.

El arte de observar, analizar, describir, sintetizar, interpretar y comunicar, están implícitos en todo proceso de representación, y ha supuesto a lo largo de la historia, toda una constante suma de reflexiones de creadores, investigadores y artistas.

Como afirma Gómez Molina (2001: 35):

Toda la historia de la enseñanza del dibujo no es más que un amplio catálogo de tautologías alrededor de visualizar y comprender los conflictos que conllevan percepción, conocimiento y actuación.

Decía Leonardo que "todos nuestros conocimientos tienen su origen en nuestras percepciones". Las reflexiones de Da Vinci nos llevan a entender la importancia de la conciencia individual en la percepción del mundo que nos rodea. Una de las claves de su genialidad reside en su capacidad para construir el conocimiento a través de la percepción de las cosas. Su capacidad para aunar pensamiento analítico (que desestructura para comprender) y pensamiento analógico (que relaciona y une las partes de un todo aparentemente desunido) fue excepcional, quedando de manifiesto su vigencia intelectual hasta nuestros días

Desde el punto de vista de las artes visuales, podríamos definir la percepción como la capacidad de captación, interpretación, análisis e integración de estímulos adquiridos por nuestros sentidos para conformar una representación de lo observado. En todos estos aspectos involucrados en el proceso, hay una parte más próxima a 
los componentes fisiológicos, como el sistema visual, y otros que han sido foco de muchas y diversas investigaciones en los campos de la psicología, la ciencia cognitiva y la neurociencia.

La importancia que tiene la actitud con que nos enfrentamos al registro gráfico de lo observado es esencial. La mirada siempre ha de estar guiada por nuestro intelecto y nuestro conocimiento. Sensibilizar la mirada implica una asociación de operaciones entre la visión, percepción y representación y una profundización en la indagación de las características generales y peculiares de lo observado.

Más allá de las consideraciones acerca de los procesos fisiológicos de la visión, en todo acto de percepción existe un sustrato de inteligencia que se relaciona con la percepción visual. Arnheim expone a cerca de la inseparabilidad de los conceptos de percepción y pensamiento:

El conjunto de las operaciones cognoscitivas llamadas pensamiento no son un privilegio de los procesos mentales situados por encima y más allá de la percepción, sino ingredientes esenciales de la percepción misma. Me refiero a operaciones tales como la exploración activa, la selección, la captación de lo esencial, la simplificación, la abstracción, el análisis y la síntesis, el completamiento, la corrección, la comparación, la solución de problemas, como también la combinación, la separación y la puesta en contexto. Estas operaciones no son prerrogativa de ninguna de las funciones mentales; son el modo en el cual tanto la mente del hombre como la del animal tratan el material cognitivo en cualquier nivel. No existe diferencia básica en este respecto entre lo que sucede cuando una persona contempla directamente el mundo y cuando se sienta con los ojos cerrados y piensa (Arnheim1993a: 20).

El autor expone en el texto citado las razones por las que sostiene la necesidad de eliminar las diferencias que muchos psicólogos hacen entre conocimiento y percepción. Arnheim incluye dentro de lo cognitivo todas las operaciones mentales implicadas de un modo inclusivo en el acto de percibir: percepción sensorial, memoria, pensamiento y aprendizaje.

Dibujar desde la observación implica un proceso de abstracción mediante el uso de un vocabulario y sintaxis gráficos con la finalidad, no de la reproducción fotográfica, sino de una representación intencionada de aquello que se quiere comunicar. Mientras dibujamos estamos reproduciendo un diálogo interactivo entre lo observado y lo dibujado, cuyo tránsito da como resultado una imagen que permanece como una referencia en el tiempo.

\section{Metodología}

\subsection{Planteamiento metodológico}

La metodología implícita en el ámbito de actuación del trabajo desarrollado se sitúa en consonancia con dos grandes corrientes: las teorías sobre la Investigación en 
la Acción aplicada al ámbito educativo y la Investigación Basada en las Artes, IBA (Art based Research-ABR- en inglés).

Estas teorías elaboradas en el ámbito de la psicología social y educativa, bien pueden servir de marco referencial en las áreas de estudio relacionadas con la enseñanza y aprendizaje de la práctica artística.

El territorio donde se desarrollamos el trabajo docente es un lugar en el que las actividades académicas deben seguir unos estándares de creatividad en consonancia con la propia filosofía de la disciplina. Debemos no dejar morir nuestro impulso innovador en los planteamientos metodológicos, porque eso generará un input de energía entre los actores del proceso que retroalimentará la ilusión y la curiosidad por el conocimiento y aprendizaje, ampliando la noción de capacidad, algo que es el pilar fundamental de todo proceso educativo.

Ken Robinson (2009) plantea muy bien esta cuestión. Sabe que la motivación es la clave para poner en movimiento la maquinaria que puede transformar la sociedad. Los educadores también, y así lo debemos intentar transmitir en el proceso de formación de nuestros estudiantes.

Por otro lado, la expresión "investigación-acción" fue acuñada por el psicólogo polaco nacionalizado estadounidense Kurt Lewin en 1946 para describir una forma de investigación que auna teoría y práctica para la consecución de sus objetivos. Ese hecho no diferenciador es clave para entender su oportunidad en el ámbito de las Bellas Artes.

En este sentido y según Elliot (1990: 15),

...la docencia no es una actividad y la investigación-sobre-la-enseñanza otra. Las estrategias docentes suponen la existencia de teorías prácticas acerca de los modos de plasmar los valores educativos en situaciones concretas, y cuando se llevan a cabo de manera reflexiva, constituyen una forma de investigación-acción. Si se considera una práctica social como la enseñanza como una actividad reflexiva, la división del trabajo entre prácticos e investigadores se desvanece.

Por otra parte, queremos enfatizar las ideas más importantes elaboradas en Investigación Basada en las Artes, sobre todo aquellas que se relacionan en mayor medida con el ámbito de la creación y educación artísticas, porque creemos que justifican claramente una forma de abordar los planteamientos metodológicos.

Así, Hernández (2008: 92), respondiendo a su propia pregunta ¿qué significa una investigación basada en las artes?, va desgranando una serie de definiciones y respuestas, entre las que destacamos las de Silverman que considera que

la finalidad de cualquier investigación es permitir acceder a lo que las personas hacen y no sólo a lo que dicen. En este sentido las artes llevan 'el hacer' al campo de investigación. (Ibid. 95);

y Speiser, que la considera como 
un método de indagación que utiliza elementos de la experiencia de las artes creativas, incluyendo el hacer arte por parte del investigador, como maneras de comprender el significado de lo que nosotros hacemos dentro de nuestra práctica y de la enseñanza (Ibid. 95).

Como sugiere Hernández,

La utilización de representaciones artísticas de carácter visual constituye uno de los principales referentes de la IBA. (Ibid. 99) .

Hernández pone como ejemplo los cuadernos de Leonardo Da Vinci donde texto e imagen se ilustran mutuamente, y propone el desarrollo en paralelo de

narrativas autónomas (textual y visual) que se complementen, entrecrucen y permitan que surjan espacios desde los que crear nuevos significados y relaciones (Ibid. 100).

Por otra parte hemos de destacar muy especialmente, en consonancia con lo sugerido por Marín, la necesidad de desarrollar el propio campo de las prácticas artísticas. En este sentido, Marín sostiene:

... Socialmente hay establecida una clara dicotomía entre Creación e Investigación: creación es el proceso de las artes visuales, e investigación es la actividad de la ciencia. Hay que desarrollar una metodología de la Investigación en Artes Visuales, no sobre las artes, que supere ese dilema... Los procesos y los resultados de la investigación en arte son fundamentalmente imágenes (cuadros, esculturas, dibujos, grabados, fotografías, etc.) (Marín 2003: 405)

Estas teorías elaboradas en el ámbito de la psicología social y educativa, han servido y sirven de marco referencial en las áreas de estudio relacionadas con la enseñanza y aprendizaje de la práctica artística y por tanto en esta investigación.

\subsection{Fases de la investigación}

\subsubsection{Identificación de dificultades}

Para identificar cuales son las dificultades o problemas más comunes, se parte de una evaluación diagnóstica que provee del material de estudio mediante dibujos de observación realizados por los estudiantes. En ellos se ponen de manifiesto un gran número de "patologías", o de problemas perceptuales y representacionales a la hora de extraer la información del modelo y plasmar el dibujo como por ejemplo: dificultad para captar la totalidad (fig. 1), construir sumando partes, afloramiento de lo simbólico, multifocalidad (fig. 2), tendencia a la frontalidad, falta de equilibrio, elección inapropiada del lugar de observación y de la distancia al tablero de dibujo, etc. 
Figura 1. Discóforo. Dibujo de estudiante de 1er curso. 100*70 cms. Carbón sobre papel. Figura 2. Cabeza de caballo

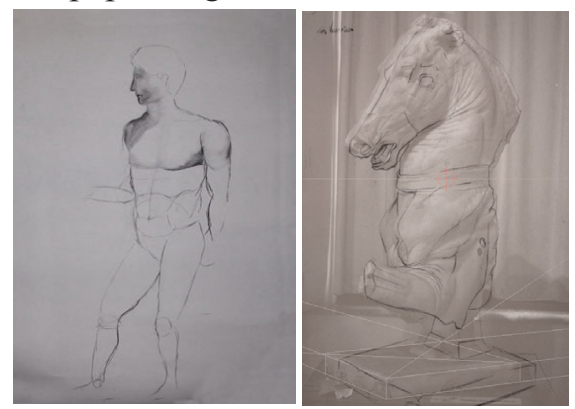

Dibujo de estudiante de 1 er curso $(100 * 70 \mathrm{cms}$. Carbón sobre papel) y estudio comparativo de posición del punto de vista mediante superposición fotográfica.

\subsubsection{Estudio de las fuentes bibliográficas clásicas y contemporáneas}

El dibujo de contorno ha sido una constante en las estrategias que los educadores y los propios artistas han usado para favorecer los procesos de percepción, profundizar en su aprendizaje, y como estímulo y acercamiento al proceso representacional. $\mathrm{Su}$ objetivo fundamental es la sensibilización y agudización de la mirada que nos centra la atención sobre lo mirado, implementando su conocimiento.

En esta fase se revisaron algunos de los autores que propusieron y desarrollaron sus métodos para la práctica del dibujo de contorno, sus modalidades, sus sugerencias y que en buena medida se han convertido en referentes de escuelas y centros de enseñanzas artísticas.

Autores como Kimon Nicolaïdes y su programa metodológico de aprendizaje basado en la utilización activa de todos los sentidos (Nicolaïdes 1960); Betty Edwards (1984), que expone a través de su conocido método basado en las teorías de las funciones de los hemisferios cerebrales el dibujo de contornos como uno de los pasos en el proceso de aprender a dibujar; Francis Ching, para quien el objetivo del dibujo de contorno es

alcanzar una correspondencia exacta entre el ojo que recorre los bordes de una forma y la mano que traza las líneas que los representan. (Ching 1999: 18).

Por otra parte, Cynthia M. Dantzic se apoya, en parte, en los ejercicios de Josef Albers para desarrollar destrezas intelectuales y manuales. Para la autora cobra especial importancia la fluidez, elegancia y plenitud de la línea en el recorrido completo de los contornos, ya sean vistos u ocultos . (Dantzic 2004: 64)

Además, se estudiaron los diferentes estilos o tipologías de línea que recogen en sus manuales Deborah Rockman (2000: 56-64), Nathan Goldstein (1973: 41-55) o Betti y Sale (1986: 108-124) 


\subsubsection{Los artistas clásicos y contemporáneos relacionados con el tema en cuestión}

Partimos del mito del origen del dibujo en la tradición occidental, como citara Plinio el Viejo en su Historia Natural, en la que recoge como la hija del alfarero Butades de Sición dibujó los bordes de la sombra del perfil de su amado sobre un pared (Stoichita 1999: 15). No dejaremos de aproximarnos a Leonardo Da Vinci, quien, en referencia al dibujo de contorno nos dice:

...el límite de una cosa es principio de otra. 2. Llamamos nada a lo que nada es parte de cosa alguna. 1. Lo que carece de límite, carece de forma. Los límites de dos cuerpos contiguos son superficies intercambiables. Los límites de los cuerpos no son parte alguna de esos mismos cuerpos. (Da Vinci 1986: 13).

Se ha estudiado la obra gráfica relacionada con el tema en cuestión de Hans Holbein, Canaletto, Vermeer e Ingres señalados entre otros por David Hockney (2001) como artistas que usaron instrumentos opticos para el registro de imágenes con un nivel de detalle y precisión desconocidos hasta entonces. La utilización del vidrio por Leonardo, el porticón de Durero, la cámara obscura descrita por Kepler e implementada posteriormente por Barbaro, la cámara lúcida y otros muchos artefactos de ayuda al Dibujo y la Pintura, gozan todos de un mismo hecho conceptual: el concepto de proyección.

Igualmente, hemos realizado un recorrido por algunos de los artistas más significativos del siglo XX, deteniéndonos en el estudio de las características de sus obras en relación con el tema tratado.

August Rodin y sus dibujos llenos de sensualidad, elegancia y erotismo, como intento de congelar un instante en el tiempo; Juan Gris quien resuelve con una solvencia extraordinaria los dibujos de contorno que llegan a mostrar una gran expresividad con el mínimo de recursos; Pablo Picasso, maestro indiscutible de la economía del lenguaje gráfico que consigue transmitir la percepción de mucho más que la realidad; Enri Matisse quien nos dice

Si confío en la mano cuando dibuja, es porque me he esforzado para que nunca se adelante a mi sentimiento (Matisse 2010: 43);

Egon Schiele, cuya fuerza y carácter se antepone a la fidelidad o corrección anatómica en sus dibujos; Giorgio Morandi que juega con las formas positivas y negativas como configuradoras de un espacio ambiguo, con los límites mediante la observación de los tonos y valores de la composición, sus sombras y sus líneas delimitadoras; Eduardo Chillida, que utilizó magistralmente la línea de contorno como elemento de un lenguaje plástico desprovisto de todo artificio, desnudo de adornos, al que eleva a una categoría poética insuperable, cuyo trazo austero modela la tridimensionalidad del modelo; Antonio López García y la búsqueda de plasmación del tiempo congelado, sus limitaciones y consecuencias. 


\subsubsection{Elementos que intervienen en los procesos de visión-percepción-representa- ción}

El proceso de percepción visual es el realizado por los órganos sensoriales y el sistema nervioso central de forma interactiva, configurando los diferentes elementos que recibimos a través de los sentidos, de la propia experiencia visual, y de la imaginación, para organizarlos, procesarlos e interpretarlos como un conjunto.

En principio, esto sucede de forma automática, es decir, abrimos los ojos y vemos todo lo que nos queda en nuestro campo de visión, y sin ser conscientes del proceso que conlleva tal actividad, generamos una imagen en nuestra conciencia. Esta imagen final es el producto de ese proceso en el que se distinguen tres fases:

- Detección, en la que se produce la captación de los estímulos visuales

- Transmisión, donde los órganos sensoriales transforman el estímulo en señales electroquímicas que son transmitidas como impulsos a través del nervio óptico al cerebro

- Procesado, en la que el cerebro interpreta los estímulos recibidos recomponiendo todos los datos para configurar una representación visual.

Sin embargo en este proceso, solo somos conscientes del resultado final del procesamiento cerebral, pero no del propio proceso realizado hasta construir la imagen representacional que se forma en nuestra conciencia.

Como fuente de conocimiento de base para enfrentarse a los procesos perceptuales y representacionales, se utilizaron en la investigación los principios y leyes de la Gestalt. Las investigaciones que sobre la visión y la percepción que a principio del siglo XX se llevaban a cabo, recurrían al tradicional método científico de análisis de los elementos de una manera aislada e individualizada para extraer conclusiones acerca del comportamiento del conjunto del que forman parte. Sin embargo, contrariamente a esta metodología, la escuela de la Gestalt establecería una serie de leyes y principios metodológicos que intentaron definir un nuevo enfoque de la investigación psicológica, considerando la estructura u organización formal de las partes, la configuración global de las partes que componen la totalidad, la clave para entender los procesos perceptivos.

Es especialmente importante el estudio de los principios de Totalidad o las propiedades de Emergencia, mediante la cual, a través de patrones visuales complejos o abstractos, tratamos de extraer representaciones visuales de un significado coherente; la Reificación, o tendencia a dotar de significado a algo; la Multiestabilidad o inversión de una región de una imagen por otra alternativa a la anterior, que utilizaran magníficamente Salvador Dalí o M. C. Escher; la Invarianza, o propiedad mediante la cual tendemos a reconocer objetos independientemente de su orientación o posición que ocupen en el espacio.

Igualmente se han estudiado y relacionado con la obra de diferentes artistas las leyes de Prägnanz o de la "buena forma"; de la relación de figura-fondo, que tan 
magistralmente utilizara Eduardo Chillida a través de sus obras, y que expresaba de manera certera cuando afirmaba que

el diálogo entre las formas, es más importante que las propias formas (Chillida 2003: 65).

En cuanto a los Principios de agrupamiento, los referentes han sido la ley de semejanza o equivalencia y algunas obras artísticas surgidas desde sus bases conceptuales (Fig. 3); la Ley de la proximidad, donde la organización perceptual de grupos de elementos se perciben asociados en

Figura 3. Vuck Cosic. Star Trek,

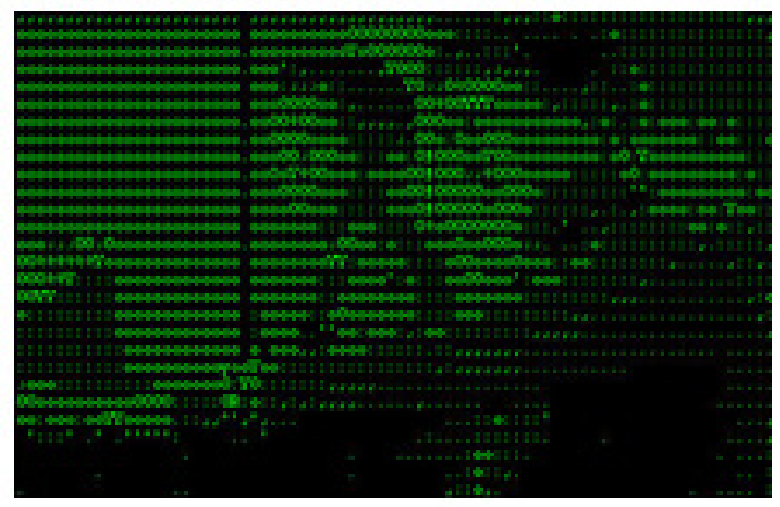

fotograma de vídeo de en ASCII.

función de su proximidad: cuanto más pequeña es la distancia entre elementos individuales, mayor es la tendencia a percibir agrupamientos que conforman una nueva unidad; la Ley de la continuidad, la cual nos conduce a percibir como conocidas las formas difícilmente reconocibles por su tamaño o distancia de observación; la Ley del destino común, en la que los elementos que se mueven (realmente o en apariencia) bajo similares criterios de velocidad, trayectoria, sentido etc, tendemos a percibirlos como pertenecientes a un mismo grupo o conformar una misma unidad, en oposición a otros que se encuentren en reposo o bajo otros criterios diferentes a los primeros; la Ley de cerramiento, que tan importante se muestra en la asimilación de los procesos de representación y que podemos resumir en la tendencia a constituir formas como un modelo completo.

\subsubsection{Uso de estrategias asociadas a tecnologías de la imagen}

David Marr, neurocientífico y psicólogo británico introduce en la década de los 70 del siglo XX un nuevo enfoque en la percepción de los objetos. Marr integra en sus estudios los conocimientos de los campos de la psicología, la neurofisiología y la inteligencia artificial, dentro de los modelos de procesamiento visual, pasando a analizar el sistema visual como si tratara de una computadora programada para 
percibir objetos o escenas. Su trabajo tuvo gran influencia en la neurociencia computacional y condujo a un resurgimiento del interés por la disciplina, alentada por los imparables avances en computación (Marr 1982).

Esta influencia de las ideas de Marr no se identificó con los adeptos de las teorías de la visión predominantes hasta entonces, que afirmaban que la construcción de un modelo interno del mundo es un requisito previo para llevar a cabo cualquier tarea visual. Los resultados de sus investigaciones contrarios a los de la neurobiología y las ciencias del comportamiento, llevaron gradualmente a un primer plano la posibilidad de que la visión no requiriese de una reconstrucción geométrica. Estos investigadores alentaron a buscar teorías alternativas, algunas de las cuales emplean conceptos y técnicas que no existían en la década de 1970, o eran desconocidas entre los estudiosos de la visión en ese momento. Estas nuevas ideas siguen hoy abriendo camino en neurociencia y visión computacional.

Marr sugirió que es posible investigar la visión en tres niveles de forma independiente y describió la visión como un procedimiento que tiene como resultado la formación de una 'matriz visual" de dos dimensiones (proyección en la retina) para una descripción del mundo en tres dimensiones. (Marr 1982: 36-38).

\subsubsection{Bordes de una imagen}

La tesis planteada por Marr tuvo una relevancia importante, entre otros muchos campos, en las técnicas de reconocimiento utilizadas en visión computacional, o en el desarrollo de software de tratamiento de imagen digital. Si analizamos las imágenes observadas desde los postulados iniciados por Marr, los bordes son significativos cambios locales en la intensidad de una imagen. Suelen producirse en el límite entre dos regiones de diferente intensidad. Por tanto podríamos decir que los bordes son las fronteras del mapa tonal de la imagen.

Los cambios en la intensidad de los píxeles pueden estar determinados por aspectos físicos o lumínicos. Entre los primeros se han de citar los límites físicos de objeto, en los que apreciamos una discontinuidad entre la intensidad y/o el color y textura superficial del objeto, y los límites superficiales que nos muestran una discontinuidad en la orientación de las superficies y/o el color de la superficie y textura.

En relación con los aspectos luminosos, hay que tener en cuenta la reflexión y dirección de la luz, las sombras propias o arrojadas sobre otros objetos y la luz reflejada. 
Figura 4. Extracción de los bordes de una imagen digital mediante software libre Gimp (en línea)

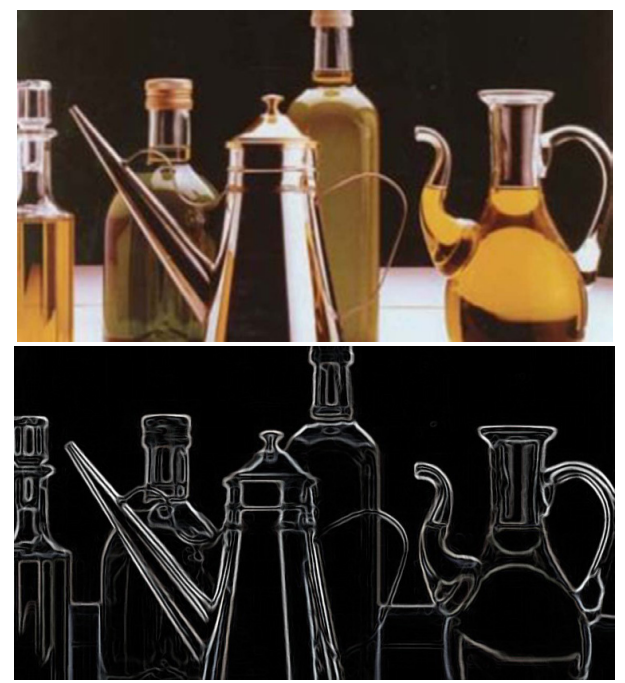

La figura 4 muestra con claridad el resultado de la búsqueda de los contornos de la escena desde el planteamiento descrito.

\section{Resultados}

Los resultados alcanzados fueron analizados desde el punto de vista del cumplimiento de los objetivos previstos, y no en relación con valores estéticos.

Como muestra, se incluyen aquí algunos dibujos que ejemplifican la comprensión de los métodos propuestos en el proceso de visión, percepción y representación por parte de los estudiantes.

Figura 5. Arriba, dibujo de un estudiante, previo a la aplicación del método propuesto. Abajo, resultado una vez aplicada la estrategia propuesta

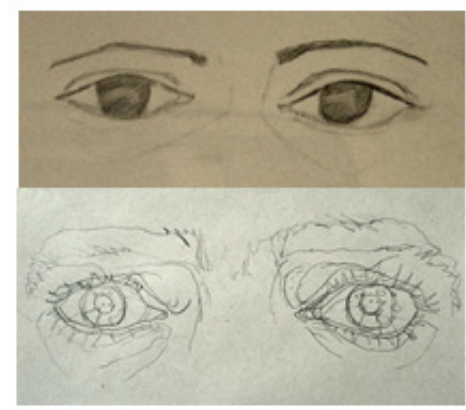


Figura 6. Dibujo de estudiante de primer curso como resultado de la estrategia propuesta

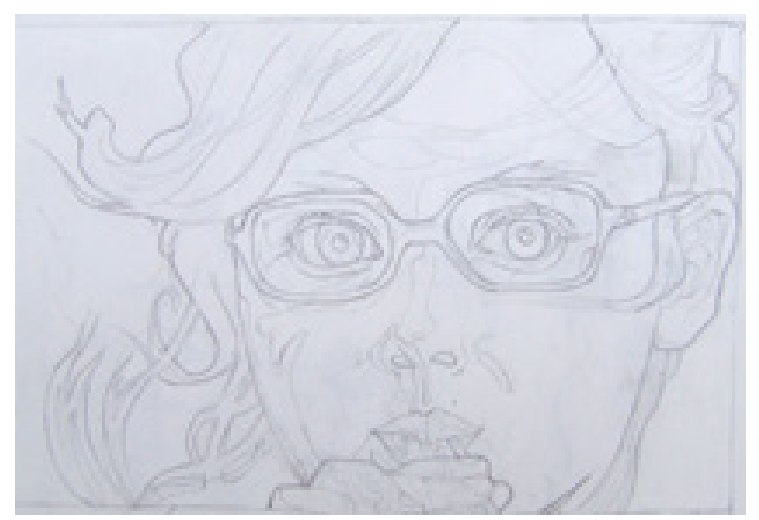

Figura 7. Dibujo de estudiante de primer curso como resultado de la estrategia propuesta

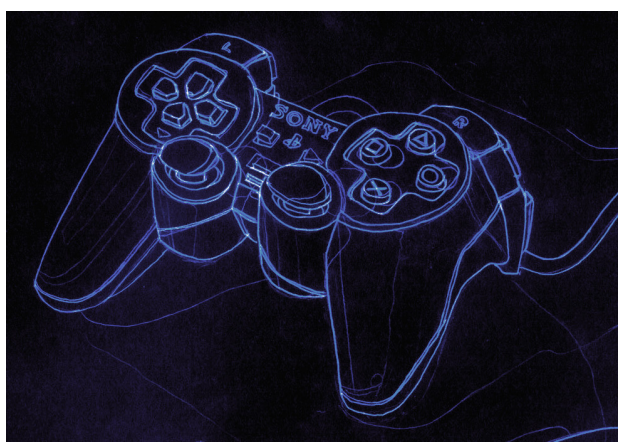

\section{Conclusiones}

Quedó demostrado que la interacción entre los ámbitos científico y artístico, entre el campo de las tecnologías visuales y el artístico, genera respuestas creativas y solventa problemas. El uso de determinadas tecnologías aplicadas, cuando se vinculan a métodos y estrategias de trabajo de observación, mejoran los resultados en la práctica experimental del dibujo.

El seguimiento del método permitió a los estudiantes adquirir la capacidad de tomar decisiones sobre qué, cómo, en qué momento mirar, y cómo extraer información relevante para la realización del dibujo y supuso un incremento significativo 
en la seguridad y abordaje de los dibujos. Se puso de manifiesto la mejora de la comprensión en su realización, y de los valores gráfico-plásticos en su ejecución.

La interacción entre el estudio de casos en la investigación aplicada a la visión artificial, especialmente en los casos de detección de bordes y la metodología procesual en la práctica del dibujo, ha ofrecido resultados muy satisfactorios, tanto en la toma de decisiones de qué dibujar, como en el resultado descriptivo y estético de los dibujos.

\section{Bibliografía}

ARNHEIM, Rudolf (1993). Consideraciones sobre la educación artística. Inglés, Fernando (trad). Barcelona. Paidós.

ARNHEIM, Rudolf(1986). El pensamiento visual. Masera, Rubén (trad). Barcelona. Paidós

BETTI, C. Y SALE, T (1986). Drawing: a contemporary approach, Florida: Holt, Rinehart and Winston, Inc., USA.

CHILLIDA, Susana, et al (2003). Elogio del horizonte: conversaciones con Chillida. Madrid.

CHING, Francis D. K., JUROSZEC, Steven P. (1999). Dibujo y Proyecto. Barcelona: Gustavo Gili.

DA VINCI, Leonardo (1986). Tratado de Pintura. González García, Ángel (Edit.). Madrid. Akal.

EDWARDS, Betty (1984). Aprender a dibujar con el lado derecho del cerebro. Ibeas, Juan M. (trad.) Madrid. Herman Blume.

ELLIOT, John (1990). La investigación-acción en educación. Madrid. Morata.

GOLDSTEIN, E. Bruce (2006). Sensación y percepción. $6^{a}$ Edic. Madrid. Paraninfo.

GOLDSTEIN, Nathan (1997). The art of the responsive drawing. 2nd. Edit. New Jersey: Prentice-Hall

GÓMEZ MOLINA, J.J., CABEZAS, L., BORDES, J. (2001). El manual de dibujo. Estrategias de su enseñanza en el siglo XX. Madrid. Cátedra.

HERNÁNDEZ HERNÁNDEZ, Fernando (2008). La Investigación basada en las artes: propuestas para repensar la investigación en educación. Educatio Siglo XXI, $n^{\circ} 26$.

HOCKNEY, David (2001). El conocimiento Secreto. Mariani, Hugo (trad.). Barcelona. Destino.

MARÍN VIADEL, Ricardo (2003). Investigación y metodologías de investigación en bellas artes. INARS. Sevilla. Universidad de Sevilla.

MARIS DANTZIC, C. (2004). Cómo dibujar. Guía completa de sus técnicas e interpretación. Tursen, S.A./H. Blume. Madrid.

MARR, David (2010). Vision: A Computational Investigartion into the Human Representation and Processing of Visual Information. Cambridge. Massachusetts, The MIT Press. 
MATISSE, H. Escritos y consideraciones sobre el arte, Paidós, Barcelona: 2010

NICOLAIDES, K (1969). The natural way to draw: a working plan for artt study Boston. Massachussets. Houghton Mifflin Harcourt.

ROCKMAN, Deborah A. (2000). The Art of Teaching Art: A Guide for Teaching and Learning the Foundations of Drawing-Based Art. New York. Oxford University Press.

STOICHITA, Victor I. (1999). Breve Historia de la Sombra. Cordech, Ana María (trad.). Madrid. Siruela.

ROBINSON, Ken (2009). El elemento: descubrir tu pasión lo cambia todo. Madrid. Grijalbo Mondadori. 